\title{
Graphene-based nano-patch antenna for terahertz radiation
}

\author{
Ignacio Llatser \\ Nanonetworking Center in Catalunya (N3Cat), Universitat Politècnica de Catalunya, Jordi Girona 1-3, Campus Nord, \\ D6-008 08034 Barcelona, Spain \\ Institute of High-Frequency and Communication Technology, Faculty of Electrical, Information and Media Engineering, \\ University of Wuppertal, Rainer-Gruenter-Str. 21, D-42119 Wuppertal, Germany \\ Christian Kremers \\ Institute of High-Frequency and Communication Technology, Faculty of Electrical, Information and Media Engineering, \\ University of Wuppertal, Rainer-Gruenter-Str. 21, D-42119 Wuppertal, Germany \\ Albert Cabellos-Aparicio \\ Nanonetworking Center in Catalunya (N3Cat), Universitat Politècnica de Catalunya, Jordi Girona 1-3, Campus Nord, \\ D6-00808034 Barcelona, Spain \\ Josep Miquel Jornet \\ Broadband Wireless Networking Laboratory, School of Electrical and Computer Engineering, Georgia Institute of Technology, \\ Atlanta, Georgia 30332, USA \\ Eduard Alarcón \\ Nanonetworking Center in Catalunya (N3Cat), Universitat Politècnica de Catalunya, Jordi Girona 1-3, Campus Nord, \\ D6-008 08034 Barcelona, Spain \\ Dmitry N. Chigrin \\ Institute of High-Frequency and Communication Technology, Faculty of Electrical, Information and Media Engineering, \\ University of Wuppertal, Rainer-Gruenter-Str. 21, D-42119 Wuppertal, Germany
}

\begin{abstract}
The scattering of terahertz radiation on a graphene-based nano-patch antenna is numerically analyzed. The extinction cross section of the nano-antenna supported by silicon and silicon dioxide substrates of different thickness are calculated. Scattering resonances in the terahertz band are identified as Fabry-Perot resonances of surface plasmon polaritons supported by the graphene film. A strong tunability of the antenna resonances via electrostatic bias is numerically demonstrated, opening perspectives to design tunable graphene-based nano-antennas. These antennas are envisaged to enable wireless communications at the nanoscale.
\end{abstract}

\section{Introduction}

Graphene has recently attracted intense attention of the research community due to its extraordinary mechanical, electronic and optical properties [1]. Being a flat monolayer of carbon atoms tightly packed in a two-dimensional honeycomb lattice, graphene allows to utilize novel physics in a plethora of potential applications, ranging from

Preprint submitted to Elsevier ultra-high-speed transistors [2] to transparent solar cells [3], meta-materials [4] and graphene plasmonics $[5,6,7,8,9]$.

One particularly promising research field is that of graphene-enabled wireless communications. Wireless communications among nanosystems, i.e., integrated systems with a size of a few micrometers, cannot be achieved by simply reducing the

August 10, 2012 


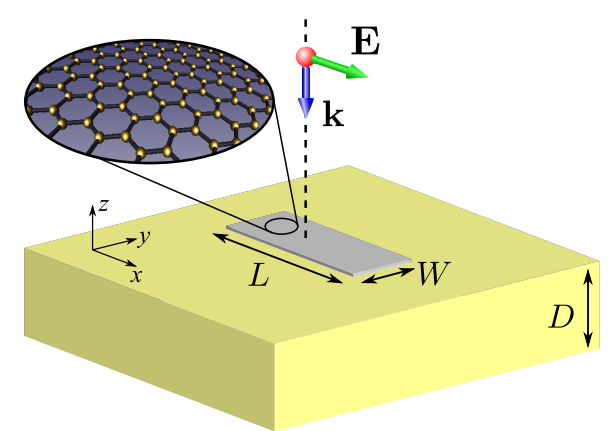

Figure 1: Sketch of the graphene-based nano-antenna under consideration.

size of classical metallic antennas down to a few micrometers. This approach presents several drawbacks, such as the low mobility of electrons in nanoscale metallic structures and especially the use of very high resonant frequencies (up to infrared and optical range), which result in a huge channel attenuation and the difficulty of implementing nanotransceivers operating at such a high frequency. For these reasons, using micrometer-size metallic antennas to implement wireless communications among nanosystems becomes unfeasible. However, owing to its ability to support surface-plasmon polaritons (SPP) [10, 11], graphene is seen as the enabling technology for this emerging field. Indeed, a graphene-based nano-patch antenna with lateral dimensions of just a few micrometers is predicted to resonate in the terahertz band $[12,13]$, at a dramatically lower frequency and with a higher radiation efficiency with respect to their metallic counterparts. In consequence, graphene-based nanoantennas are envisaged to enable wireless communications among nanosystems [14].

In this work, we present a systematic numerical analysis of the terahertz radiation scattering on a graphene-based nano-patch antenna (Figure 1). A graphene rectangular patch with length $L$ and width $W$ supported by a dielectric substrate of thickness $D$ is illuminated by a plane wave linearly polarized along the patch length. The associated scattering problem is then solved numerically, and the extinction, absorption and scattering cross sections are analysed.

The remainder of this paper is organized as follows. In Section 2, the expression used to model the electric conductivity of graphene is presented. In Section 3, two different approaches to model a graphene patch are described and compared. Sec-

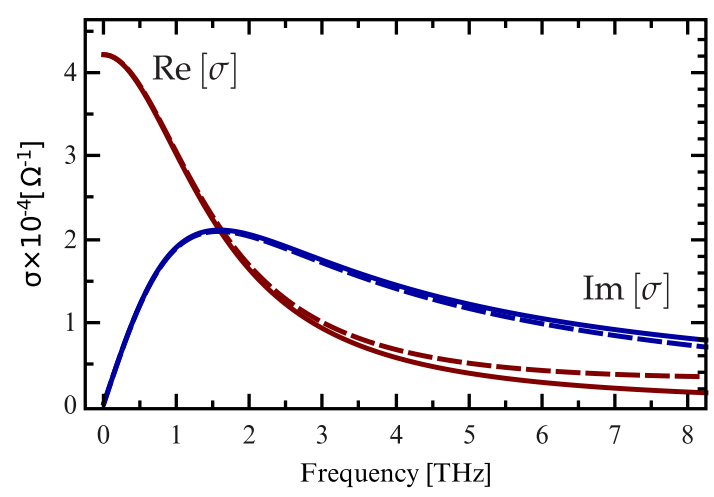

Figure 2: Real and imaginary part of the total conductivity (solid lines) and the intraband conductivity (dashed lines) at room temperature $(T=300 \mathrm{~K})$ and zero electrostatic bias $\left(\mu_{c}=0 \mathrm{eV}\right)$

tion 4 explores the dependence of the graphenebased nano-antenna cross sections as a function of several pameters, such as the antenna dimensions or the chemical potential. Finally, Section 6 concludes the paper.

\section{Graphene conductivity}

Graphene-based nano-patch antennas are envisaged to have a length and width in the order of a few micrometers $[12,13]$. Since it has been experimentally demonstrated that edge effects on the graphene conductivity only appear in structures with lateral dimensions considerably smaller than $100 \mathrm{~nm} \mathrm{[15],} \mathrm{in} \mathrm{this} \mathrm{analysis} \mathrm{we} \mathrm{will} \mathrm{disre-}$ gard the edge effects and will use the electrical conductivity model developed for infinite graphene sheets $[10,11]$.

The surface conductivity of an infinite graphene film can be calculated by means of the Kubo formalism $[10,11]$. Within the random-phase approximation, the surface conductivity can be represented in a local form with the Drude-like intraband contribution

$$
\sigma(\omega)=\frac{2 e^{2}}{\pi \hbar} \frac{k_{B} T}{\hbar} \ln \left[2 \cosh \left[\frac{\mu_{c}}{2 k_{B} T}\right]\right] \frac{i}{\omega+i \tau^{-1}},
$$

and interband contribution given by

$\sigma_{i}(\omega)=\frac{e^{2}}{4 \hbar}\left(H\left(\frac{\omega}{2}\right)+\mathrm{i} \frac{4 \omega}{\pi} \int_{0}^{\infty} \mathrm{d} \epsilon \frac{H(\epsilon)-H(\omega / 2)}{\omega^{2}-4 \epsilon^{2}}\right)$.

Here $\tau=10^{-13} \mathrm{~s}$ is the relaxation time, $T$ is the temperature, $\mu_{c}$ is the chemical potential and $H(\epsilon)$ 
is defined as

$$
H(\epsilon)=\frac{\sinh \left(\hbar \epsilon / k_{B} T\right)}{\cosh \left(\mu_{c} / k_{B} T\right)+\cosh \left(\hbar \epsilon / k_{B} T\right)} .
$$

In the frequency region of interest (below $5 \mathrm{THz}$ ), the intraband contribution (1) dominates [16]. This can be seen in figure 2, where the frequency dependence of the real and imaginary part of the intraband conductivity $\sigma$ and the total conductivity $\sigma_{\text {total }}=\sigma+\sigma_{i}$ are compared at room temperature $(T=300 \mathrm{~K})$ and zero electrostatic bias $\left(\mu_{c}=0 \mathrm{eV}\right)$. In the following, we neglect the interband conductivity contribution and assume the conductivity of the graphene patch to be purely intraband one.

\section{Numerical methods}

In order to find the electromagnetic field scattered by a graphene structure, it is necessary to couple the phenomenological model of graphene conductivity with Maxwell's equations. The major challenge here is to model an infinitesimally thin graphene layer using a finite-size discretization of space typical for numerical calculations.

There are two main methods that can be used to model a graphene sheet. The first technique consists in approximating a graphene layer by an equivalent thin slab with a small, but finite, width. The propagation of the electromagnetic fields within the slab is modeled by assigning to it a normalized effective conductivity $[4,7]$

$$
\overleftrightarrow{\sigma}=\left(\begin{array}{ccc}
\sigma / \Delta & 0 & 0 \\
0 & \sigma / \Delta & 0 \\
0 & 0 & 0
\end{array}\right)
$$

where $\Delta$ is the thickness of the equivalent slab and the graphene sheet is located in the $\mathrm{x}-\mathrm{y}$ plane. The main drawback of this method is that a realistic model of graphene will have a length $L$ much larger than its thickness $\Delta$, resulting in a very high aspect ratio $(L / \Delta \sim 1000)$. The numerical computation of the electromagnetic fields in such a structure will therefore require a very high mesh density, leading to a high computational cost.

As an alternative to treat this problem with lower computational costs, the graphene sheet can be modeled as an equivalent impedance surface [13, 17]. The surface impedance $Z_{s}$ connects the tangential component of the electric field on the surface with the electric surface current, $\left.\mathbf{E}_{\tau}\right|_{z=0}=Z_{s} \mathbf{J}_{\text {surf }}$. Taking into account that the current induced in the

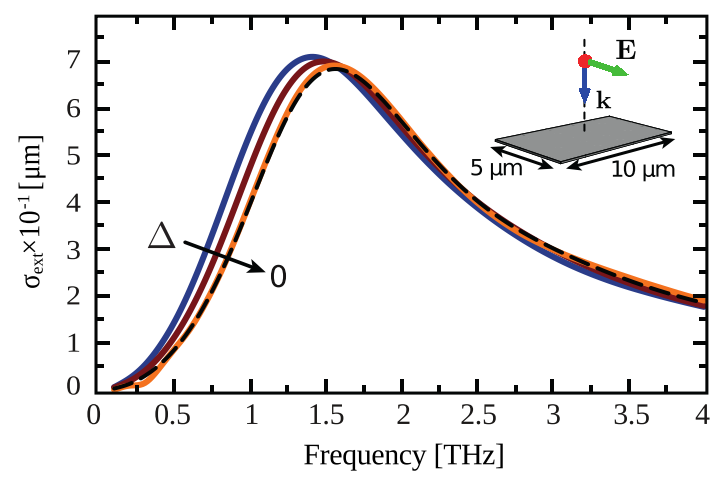

Figure 3: Extinction cross section per unit width of a $10 \mu \mathrm{m}$ wide graphene patch with length $L=5 \mu \mathrm{m}$. Results of the surface impedance model (dashed black line) and the equivalent slab model (solid lines ) are shown at room temperature $(T=300 \mathrm{~K})$ and zero electrostatic bias $\left(\mu_{c}=0 \mathrm{eV}\right)$. The thicknesses of the equivalent graphene slab $\Delta$ are $500 \mathrm{~nm}$, $200 \mathrm{~nm}$ and $5 \mathrm{~nm}$, from left to right.

graphene layer is purely superficial and it is related to the tangential component of the electric field via the surface conductivity $\sigma$ as $\mathbf{J}_{\text {surf }}=\left.\sigma \mathbf{E}_{\tau}\right|_{z=0}$, one can define the boundary conditions at the graphene interface

$$
\hat{\mathbf{n}} \times\left[\left.\mathbf{H}\right|_{z=+0}-\left.\mathbf{H}\right|_{z=-0}\right]=\mathbf{J}_{\mathrm{surf}}=\left.\frac{1}{Z_{s}} \mathbf{E}_{\tau}\right|_{z=0}
$$

where $Z_{s}=1 / \sigma$ is the equivalent surface impedance of the graphene. The boundary conditions (3) fully determine the electromagnetic problem and can be solved numerically using a computational scheme of choice.

Figure 3 contains a comparison between the equivalent slab model and the surface impedance model. In all numerical calculations the method of moments with surface equivalence principle [18] has been employed. The solid lines show the extinction cross section (see Sec.4 for details) of the antenna as a function of frequency when the antenna is modeled as a thin slab, with an effective conductivity as defined in (2), for different antenna thicknesses: $500 \mathrm{~nm}, 200 \mathrm{~nm}$ and $5 \mathrm{~nm}$, from left to right. The dashed line corresponds to the surface impedance model. One can see that the equivalent slab model converges to the surface impedance model as the equivalent slab thickness is reduced, while simultaneously requiring denser mesh (higher computational costs) for smaller equivalent thickness. In what follows the surface impedance model is used to numerically characterize the graphene patch due 
to its accuracy and efficiency.

\section{Scattering properties}

In order to study the performance of a graphenebased nano-patch antenna, it is interesting to investigate the scattering, absorption and extinction cross sections of a graphene patch. The scattering (absorption) cross section is defined as a ratio of the scattered (dissipated) power to the incident power, namely

$$
\sigma_{\text {scat }}=\frac{\oint_{S} d^{2} r \mathbf{S}_{s} \cdot \mathbf{n}}{\left|\mathbf{S}_{\text {inc }}\right|}
$$

and

$$
\sigma_{a b s}=\frac{\oint_{S} d^{2} r \mathbf{S} \cdot \mathbf{n}}{\left|\mathbf{S}_{i n c}\right|}
$$

Here the surface integration is performed over a surface enclosing the graphene antenna. $\mathbf{n}$ is the surface normal. $\mathbf{S}, \mathbf{S}_{s}$ and $\mathbf{S}_{\text {inc }}$ are the Poynting vectors of the total, scattered and incident fields, respectively. The extinction cross section is given by the sum of the scattering and the absorption cross sections

$$
\sigma_{e x t}=\sigma_{s c a t}+\sigma_{a b s} .
$$

The calculated scattering and absorption cross sections of a graphene patch with length $L=1 \mu \mathrm{m}$ and width $W=100 \mu \mathrm{m}$ normalized to the patch geometrical area are shown in figure 4 . The antenna is supported by an infinite silicon substrate with dielectric constant $\varepsilon=11.9$. Room temperature $(T=300 \mathrm{~K})$ and zero electrostatic bias $\left(\mu_{c}=0 \mathrm{eV}\right)$ are assumed. The interaction of the terahertz radiation with the antenna is dominated by the absorption, with the scattering being three orders of magnitude weaker due to the large wavelength mismatch between the electromagnetic excitation in the graphene layer and in the far-field. The total extinction cross section is equal to a few percents of the graphene area and demonstrates a clear resonant character. The obtained absorption cross sections are consistent with experimental results reported for graphene micro-ribbon arrays [19].

In order to understand the resonant behavior, we consider a simple Fabry-Perot (FP) model for the graphene patch. An infinite graphene layer placed on the air-dielectric interface supports transversemagnetic (TM) SPP waves with a dispersion rela-

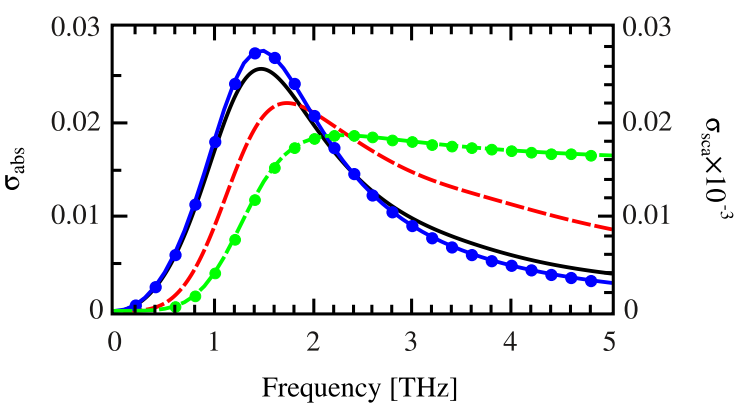

Figure 4: The absorption (black line) and scattering (dashed red line) cross sections of a graphene antenna on an infinite silicon substrate, obtained using numerical simulations, as compared with the absorption (blue line with dots) and scattering (dashed green line with dots) cross sections obtained using the Fabry-Perot model.

tion given by [5]

$$
\frac{1}{\sqrt{\beta^{2}-\frac{\omega^{2}}{c^{2}}}}+\frac{\varepsilon}{\sqrt{\beta^{2}-\varepsilon \frac{\omega^{2}}{c^{2}}}}=-\mathrm{i} \frac{\sigma(\omega)}{\omega \varepsilon_{0}},
$$

where $\beta$ and $\omega$ are the wave-vector and frequency of the SPP wave and $\varepsilon$ is the dielectric constant of the substrate. While the air-dielectric interface does not support SPP waves, a termination of the graphene film acts as a mirror and a FP type resonator can be realized when the following condition is satisfied

$$
L_{\mathrm{eff}}=L+2 \delta L=m \frac{\lambda}{2}=m \frac{\pi}{\beta} .
$$

Here $\lambda$ is the SPP wavelength, $m$ is an integer, $L_{\text {eff }}$ is the effective resonator length and $\delta L$ is a measure of the field penetration outside the graphene patch. Solving the dispersion relation (7) with the FP condition (8) for a given effective resonator length $L_{\text {eff }}$ results in a set of $m$ complex frequencies $\omega_{m}$ (resonator modes). The coupling of the incident radiation with those modes leads to resonances in the extinction spectra. Taking into account that the modes of the resonator are orthogonal, one can model them as a set of independent driven harmonic oscillators with angular frequencies $\omega_{0 m}=\sqrt{\left(\operatorname{Re} \omega_{m}\right)^{2}+\left(\operatorname{Im} \omega_{m}\right)^{2}}$ and damping rates $\gamma_{m}=-2 \operatorname{Im} \omega_{m}$. Introducing the dipole polarizabilities of the oscillators $\alpha_{m}$ as

$$
\vec{\mu}_{m}=\frac{f_{m}}{\omega_{0 m}^{2}-\omega^{2}-\mathrm{i} \gamma_{m} \omega} \mathbf{E}_{i n c}=\alpha_{m} \mathbf{E}_{i n c},
$$


with $f_{m}$ being the oscillator strength, the scattering and absorption cross sections of the graphene antenna can be calculated as a sum of the normalized scattered and dissipated power of individual oscillators [20], namely

$$
\sigma_{s c a}=\frac{\omega^{4}}{6 \pi \varepsilon_{0}^{2} c^{4}} \sum_{m=1}^{N}\left|\alpha_{m}\right|^{2}
$$

and

$$
\sigma_{a b s}=\frac{\omega}{\varepsilon_{0} c} \sum_{m=1}^{N} \operatorname{Im} \alpha_{m} .
$$

In figure 4 , the absorption and scattering cross sections obtained with the FP model are compared with the results of a direct numerical simulation. The effective length of the FP resonator is set to $L_{\text {eff }}=1.36 \mu \mathrm{m}$, where the penetration length $\delta L=0.18 \mu \mathrm{m}$ has been estimated based on the numerical simulations. With the oscillator strength $f_{m}$ used as a fit parameter, a reasonable agreement with numerical results can be achieved even if only the first FP mode $\left(f_{1}=0.073\right)$ is taken into account.

While the operation of the graphene patch antenna is based on the SPP resonances, it is important to analyze the SPP properties. In figure 5 the SPP wavelength is shown as a function of the frequency. One can see that in the terahertz frequency range the wavelength of SPP is of the order of a few micrometers, matching the expected size of envisaged graphene-based nano-antennas. In the same time the propagation length, shown in Fig. 5 in units of the corresponding SPP wavelength, is relative small staying below one SPP wavelength. This result is consistent with the SPP behavior observed at infrared frequencies [21]. However, since a resonant graphene patch antenna will have dimensions of approximately half the SPP wavelength, the sub-wavelength propagation ohmic losses in the graphene sheet are not expected to hamper the performance of graphene antennas considerably.

\section{Scattering tuning}

The spectral position of the SPP resonance can be adjusted by an appropriate choice of the resonator length and width. The dependence of the resonant frequency on the resonator length, calculated using equivalent surface impedance method, is shown in figure 6 for different resonator widths.

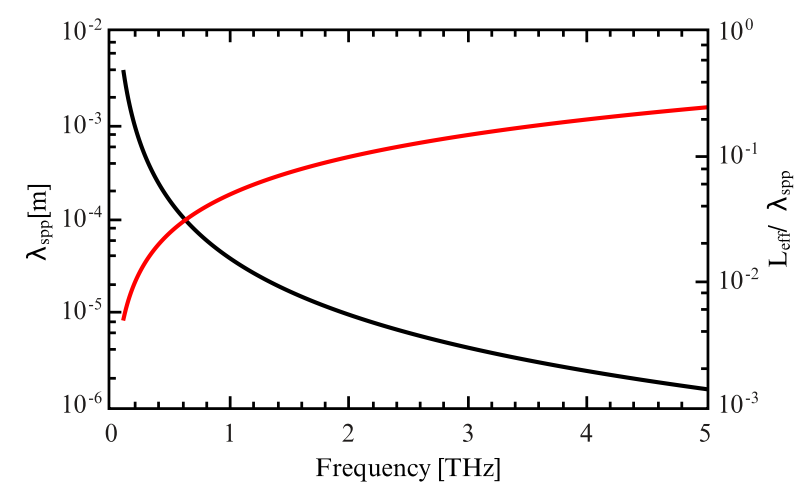

Figure 5: Logarithmic plot of the plasmon wavelength (black line, left vertical axis) and the ratio of the propagation length to the plasmon wavelength (red line, right vertical axis) in a free-standing graphene layer as a function of frequency. The frequency ranges from 0.1 to $5 \mathrm{THz}$.

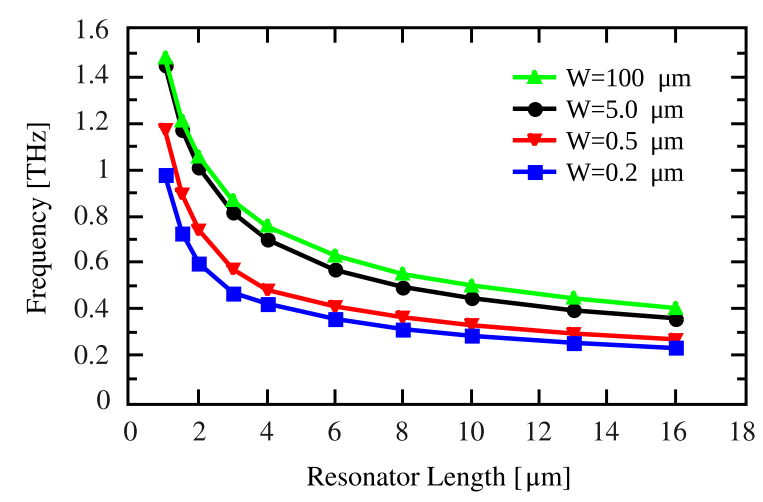

Figure 6: Dependence of the first resonant frequency of the graphene antenna on its length, $L$, for different widths, $W=$ $100 \mu \mathrm{m}, W=5 \mu \mathrm{m}, W=0.5 \mu \mathrm{m}$ and $W=0.2 \mu \mathrm{m}$.

The results correspond to a graphene patch on infinite silicon substrate at room temperature and zero electrostatic bias. A wide frequency range in the terahertz band can be covered by choosing antenna dimensions. For a given length, antennas with smaller width possess resonance at lower frequencies. This effect might be attributed to the higher confinement of surface plasmons in a narrow graphene patch, which in turn leads to higher effective permittivity and lower resonance frequency. In the same time, the resonance shifts towards higher frequencies for shorter antennas, in full agreement with the resonance condition (8).

The dielectric constant and thickness of the substrate influence both the spectral position and magnitude of the resonance. In figure 7 (top), the extinction cross section of the graphene antenna with length $L=1 \mu \mathrm{m}$ and width $W=0.5 \mu \mathrm{m}$ 

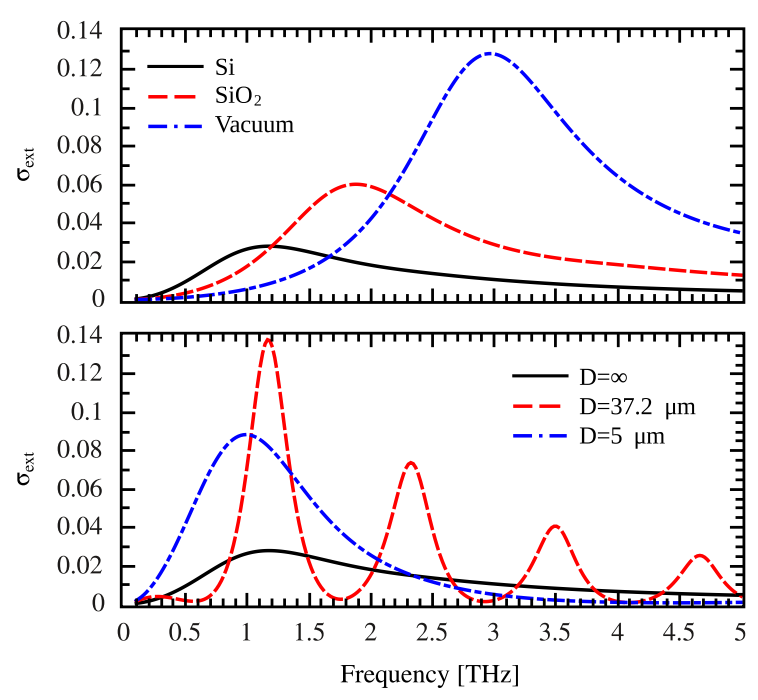

Figure 7: Top: Normalized extinction cross section of the graphene antenna placed on different substrates, silicon ( $\varepsilon=11.9$ ), silica $(\varepsilon=4.0$ ) and vacuum (left to right). Bottom: Normalized extinction cross section for different thickness of silicon substrates, $D=\infty$ (solid), $D=37.5 \mu \mathrm{m}$ (dashed) and $D=5 \mu \mathrm{m}$ (dashed-dotted).

supported by different infinite substrates is shown. As expected from the SPP dispersion relation (7), an increase of the dielectric constant of the substrate shifts the resonance towards lower frequencies, while simultaneously reducing the extinction efficiency. For a silicon substrate, a fourfold reduction of the total extinction cross section can be observed in comparison to the antenna suspended in air. One can partially compensate this reduction by adjusting the substrate thickness. In figure 7 (bottom), the extinction cross section is shown for the antenna on silicon substrate with different thicknesses. Due to constructive interference in the substrate, the extinction cross section can restore its value corresponding to the antenna in air. In particular, if the FP resonance of the substrate coincides with the one of the antenna $(D=37.5 \mu \mathrm{m})$, a fivefold enhancement of the extinction cross section can be achieved.

The strong dependence of the graphene conductivity on the chemical potential $\mu_{c}$ opens the possibility to tune the antenna resonant frequency. In figure 8 , the extinction cross section of the graphene antenna for different values of $\mu_{c}$ is shown. A chemical potential of $0.2 \mathrm{eV}$ results in the resonant frequency shift of $1.4 \mathrm{THz}$, while corresponding to a bias field below $1 \mathrm{~V} / \mathrm{nm}$ which is experimentally feasible and can be achieved with a bias voltage well
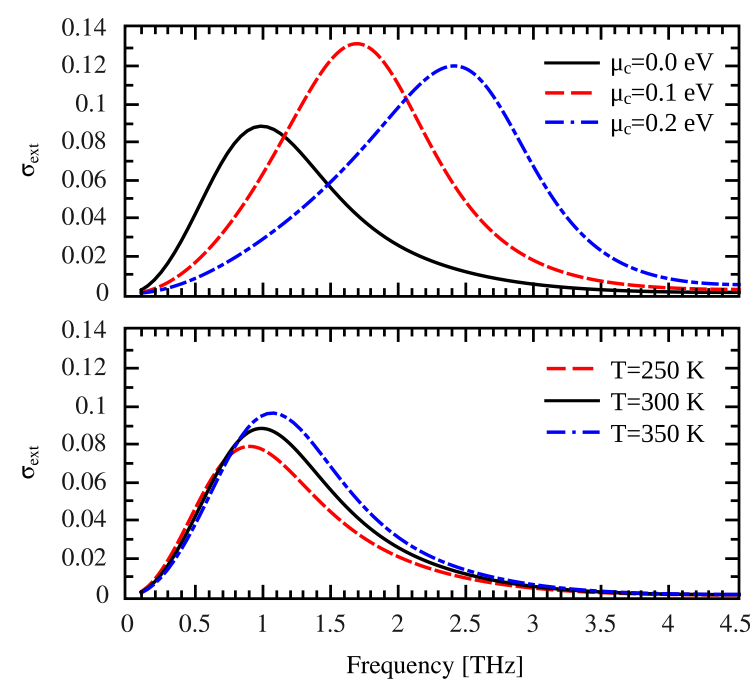

Figure 8: Dependence of the antenna resonant frequency on the electrostatic bias at $T=300 \mathrm{~K}$ (top) and on the temperature at $\mu_{c}=0 \mathrm{eV}$ (bottom). $L=1 \mu \mathrm{m}, W=0.5 \mu \mathrm{m}$, $D=\infty$ and $\varepsilon=11.9$.

below 100 volts [11]. This could enable a highly tunable antenna in the terahertz band. At the same time, such an antenna would also possess high temperature stability. For the considered structure, a $100 \mathrm{~K}$ temperature variation results in a resonant frequency shift of only $0.15 \mathrm{THz}$ (Figure 8).

\section{Conclusions}

To conclude, we have analyzed the radiation scattering on a rectangular graphene-based nanopatch antenna. Due to the propagation of surfaceplasmon polariton waves in graphene, the antenna shows a resonant behavior in the terahertz frequency band. Moreover, the simulation results demonstrate that such a structured graphene film has the potential to be used as a tunable terahertz antenna. Indeed, we have observed that the antenna resonant frequency can be tuned by changing the substrate material or size, or by applying an external electrostatic bias.

\section{Acknowledgements}

This work has been partially supported by the FPU grant of the Spanish Ministry of Education and by the European Science Foundation through an European Young Investigator Award. 


\section{References}

[1] A.K. Geim, and K.S. Novoselov, "The rise of graphene," Nat. Mater. 6, 183-191 (2007).

[2] F. Schwierz, "Graphene transistors," Nat. Nanotechnol., 5, 487-496 (2010).

[3] X. Wang, L. Zhi, and K. Müllen, "Transparent, Conductive Graphene Electrodes for Dye-Sensitized Solar Cells," Nano Lett. 8, 323-327 (2008).

[4] A. Vakil, and N. Engheta, "Transformation Optics Using Graphene," Science 332, 1291-1294 (2011).

[5] M. Jablan, H. Buljan, and M. Soljačić, "Plasmonics in graphene at infrared frequencies," Phys. Rev. B, 80, 1-7 (2009).

[6] F. Schedin, E. Lidorikis, A. Lombardo, V.G. Kravets, A.K. Geim, A.N. Grigrenko, K.S. Novoselov and A.C. Ferrari, "Surface-Enhanced Raman Spectroscopy of Graphene", ACS Nano 4, 5617-5626 (2010).

[7] F.H.L. Koppens, D.E. Chang, and F.J. García de Abajo, "Graphene Plasmonics: A Platform for Strong Light-Matter Interactions.," Nano Lett., 11, 3370-3377 (2011).

[8] Z. Fei, G.O. Andreev, W. Bao, L.M. Zhang, A.S. McLeod, C. Wang, M.K. Stewart, Z. Zhao, G. Dominguez, M. Thiemens, M.M. Fogler, M.J. Tauber, A.H. Castro-Neto, C.N. Lau, F. Keilmann and D.N. Basov, "Infrared Nanoscopy of Dirac Plasmons at the Graphene-SiO2 Interface", Nano Letters 11, 4701-4705 (2011).

[9] B.D. Dawson, M.S. Lodge, N. Nader Esfahani, R.E. Peale, M. Ishigami, "Measurement of plasmon dispersion in graphene: tunable graphene plasmonics", American Physical Society, APS March Meeting (2012).

[10] L. Falkovsky, and S. Pershoguba, "Optical far-infrared properties of a graphene monolayer and multilayer," Phys. Rev. B 76, 1-4 (2007).

[11] G.W. Hanson, "Dyadic Green's Functions for an Anisotropic, Non-Local Model of Biased Graphene," IEEE Trans. Ant. Prop. 56, 747-757 (2008).

[12] J.M. Jornet, and I.F. Akyildiz, "Graphene-based Nanoantennas for Electromagnetic Nanocommunications in the Terahertz Band," in Proc. 4th European Conf. on Antennas and Propagation, (2010).

[13] I. Llatser, C. Kremers, A. Cabellos-Aparicio, J.M. Jornet, E. Alarcon, and D.N. Chigrin, "Scattering of Terahertz Radiation on a Graphene-based Nano-antenna," AIP Conf. Proc., 1398, 144-147 (2011).

[14] I.F. Akyildiz, and J.M. Jornet, "Electromagnetic Wireless Nanosensor Networks," Nano Communication Networks, 1, 3-19 (2010).

[15] M. Y. Han, B. Oezyilmaz, Y. Zhang and P. Kim, "Energy band gap engineering of graphene nanoribbons", Phys. Rev. Lett. 98, 206805 (2007).

[16] S.A. Mikhailov, "Non-linear electromagnetic response of graphene", Europhys. Lett. 79 (2007) 27002.

[17] P.-Y. Chen and A. Alu, "Atomically Thin Surface Cloak Using Graphene Monolayers," ACS Nano, 5, 5855-5863 (2011).

[18] EM Software and Systems, FEKO, www.feko.info.

[19] L. Ju, B. Geng, J. Horng, C. Girit, M. Martin, Z. Hao, H.A. Bechtel, X. Liang, A. Zettl, Y.R. Shen, and F. Wang, "Graphene plasmonics for tunable terahertz metamaterials," Nature Nanotechnology, 6, 630-634 (2011).
[20] L. Novotny, and B. Hecht, Principles Of Nano-Optics (Cambridge University Press, Cambridge, 2006).

[21] P. Tassin, T. Koschny, M. Kafesaki, and C.M. Soukoulis, "A comparison of graphene, superconductors and metals as conductors for metamaterials and plasmonics," Nature Photonics 6, 259-264 (2012). 